Article

\title{
Human Bodily Asymmetry Relates to Behavioral Lateralization and May not Reliably Reflect Developmental Instability
}

\author{
Stefan Van Dongen $\mathbb{1}^{(1)}$ \\ Evolutionary Ecology Group, Department of Biology, University of Antwerp, 2610 Antwerp, Belgium; \\ Stefan.vandongen@uantwerpen.be; Tel.: +32-3-2653336
}

Received: 23 January 2018; Accepted: 12 April 2018; Published: 18 April 2018

check for updates

\begin{abstract}
Background: The link between behavioral lateralization and bodily asymmetry in humans is studied to investigate the reliability of fluctuating asymmetry as a measure of developmental instability; (2) Methods: Morphological asymmetries of arms and legs, obtained from 3D body scans, were correlated with different measures of behavioral lateralization; (3) Results: Observed associations were in the directions expected, showing that more asymmetric use of the body increases asymmetry, especially in the arms, and more symmetric body use appears to have a symmetrizing effect; and (4) Conclusions: The results presented here question the suitability of human bodily asymmetry in arms and legs—or at least part of them—as a measure of developmental instability. There is a need for future research that identifies regions of the body that are not affected by behavioral lateralization and can reliably reflect developmental instability.
\end{abstract}

Keywords: human; directional asymmetry; fluctuating asymmetry; developmental instability; behavioral lateralization

\section{Introduction}

Developmental instability (DI), i.e., the inability of an organism to buffer its development against random perturbations, has been studied intensively in an evolutionary and ecological context in a variety or organisms including humans. The degree of directionally random asymmetry of bilateral traits is a common measurement with which to estimate DI. This so-called fluctuating asymmetry (FA) is assumed to originate from the accumulation of small developmental perturbations that occur independently on both sides [1,2]. The suitability of FA as a measure of DI, however, depends on a number of specific assumptions that may not always be realistic. The central argument why FA is expected to reflect DI is that both sides represent independent identical developmental events in which both sides are under the control of identical genetic and environmental conditions [1-3]. Under such an ideal scenario, both sides have the same expected or 'target' phenotype and should develop into perfect symmetry if development is purely deterministic. In the presence of random developmental perturbations, subtle asymmetries emerge. Things become more complicated when the 'target' phenotype differs between sides, resulting in a systematic bias with regard to one direction on average for the population level. This directional asymmetry (DA) appears to be widespread in a variety of organisms and traits [2]. Statistically, it is still possible to separate this systematic asymmetry from the random component by subtracting the average population level asymmetry from the individual asymmetries. As long as the population is homogeneous in terms of its DA, this approach can yield valid estimates of the random stochastic component of development. Yet, if individuals also differ in their degree of DA, it is no longer possible to separate FA from DA, invalidating the use of FA as a measure of DI. Given the generality of DA across many species and the existence of behavioral 
lateralization in many vertebrates and invertebrates, the study of DA, and specifically heterogeneity of asymmetry among individuals due to behavioral lateralization, is crucial to evaluate if FA can be expected to signal DI.

Behavioral lateralization, commonly observed in vertebrates, results in DA, and the degree of DA in skulls and extremities has been invoked to find indications of behavioral lateralization, in which differences in mechanical loading between sides lead to deviations from perfect symmetry [4-6]. In humans, differential use also leads to asymmetric development, in which playing tennis affects muscles and bones in the dominant arm [7], heavy working conditions coincide with higher DA [8], and hand asymmetry correlates with handedness [9]. To complicate matters even more, cross-asymmetry across upper and lower limbs has been observed [4], suggesting that the asymmetric use of upper limbs affects the asymmetry of lower limbs as well, yet in opposite direction. In sum, there is substantial evidence of the effects of lateralization on asymmetry. Since handedness and the asymmetric use of extremities varies among individuals, a simple correction for it based on population based DA is incorrect, invalidating the use of arm (and leg) FA as a measure of DI. Nevertheless, FA of extremities continues to be used in the human FA literature, largely ignoring this aspect $[6,10]$. This paper contributes to this literature by reporting on associations between bodily asymmetry and measures of symmetric and asymmetric use of arms and legs. Average bodily asymmetry is expected to relate positively with asymmetric body use and negatively with symmetric body use. With respect to single trait asymmetries, arms and legs can be expected to show increased right biased asymmetry with increasing right biased handedness and footedness, while leg asymmetry is expected to be left-biased in right handed individuals.

\section{Materials and Methods}

Study sample: We studied asymmetry of extremities on the basis of 3D body scans and questionnaires of 107 students (42 men, 69 women) from the University of Antwerp, aged between 18 and 30 (mean $=22.8$, standard deviation $=4.8$ ). Students were contacted by email and invited to take part in this study after being informed about the procedures and signing an informed consent form. Data were collected in 2010 and 2011. Some participants agreed to additional visits to obtain repeated scans required for the analysis of measurement error. In total, 252 scans were collected, in which 47 individuals were scanned only once, 8 were scanned twice, and 41 students were scanned three times; there were two students with 4 scans, four with 5 scans, two with 6 scans, two with 7 scans, and 1 participant was scanned 12 times.

Morphological measurements and questionnaires: Measurements on upper and lower extremities were obtained from the $[\mathrm{TC}]^{2}$ body scanner (www.tc2.com), a 24-camera optical system with submillimeter accuracy [11]. Traits used are given in Table 1. Participants were asked to rate their degree of handedness and footedness on a 1-9 scale (in which a score of 1 reflects extreme left handedness/footedness and a score of 9 extreme right handedness/footedness), as well as the weekly number of hours performing 'symmetric' (like jogging, fitness, biking, swimming) and 'asymmetric' (like tennis, badminton, table tennis) sports. Frequency distributions of handedness, footedness, and hours of symmetric and asymmetric sports are provided in Figure 1 to illustrate the between-individual variation.

Statistical analyses: Measurements from the $[\mathrm{TC}]^{2}$ body scanner were analyzed using a mixed regression model [12], with trait value as response variable and side as continuous covariate (left $=-0.5$ and right $=0.5$ ). Individual and side-by-individual interactions were treated as random effects. Due to scans being prone to small scanning errors and movements of individuals, some scans did not lead to trait measurements or yield outliers (observations with a scaled residual $>3$ or $<-3$ ) that were removed for further analyses. The number of such outliers for each trait was reported. Directional asymmetry was tested through the significance of the average slope, while the amount of real FA was estimated through the variance component of the side-by-individual interaction (i.e., variation in slopes). Normality was tested for each trait using the Shapiro-Wilks test, and kurtosis was estimated for 
each trait, as antisymmetry would cause platykurtosis. Measurement error (ME) was estimated by the residual variance [12]. All further analyses were based on traits with $\mathrm{ME}<\mathrm{FA}$ (i.e., selection criterion S1 in Table 2). For those traits, we tested for positive correlations between the unsigned asymmetry (absolute value of left minus right trait value) and trait size, and divided unsigned asymmetries by trait size to correct for significant size dependency.
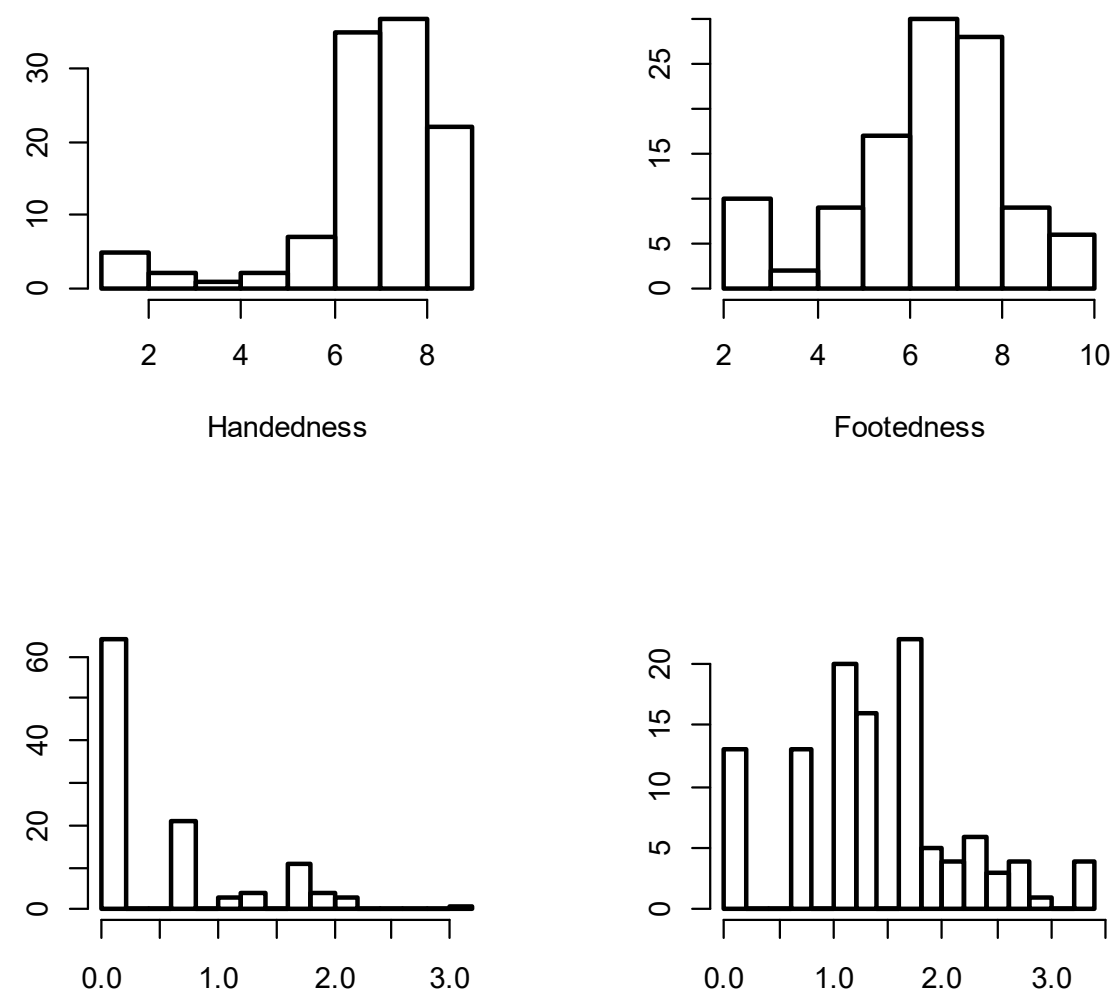

Hours asymmetric sports (log-transformed)

Hours symmetric sports (log-transformed)

Figure 1. Frequency distributions of handedness, footedness, and number of hours per week performing asymmetric and symmetric sports.

Table 1. Overview of tests for directional asymmetry (DA), estimates of variation in fluctuating asymmetry (FA), measurement error (ME), repeatability (\%FA), number of scan errors, and removed outliers. Statistically significant DA is indicated as follows: ${ }^{*} p<0.05 ;{ }^{* *} p<0.01 ;{ }^{* * *} p<0.001$.

\begin{tabular}{|c|c|c|c|c|c|c|}
\hline \multirow{2}{*}{ Trait } & \multirow{2}{*}{$\begin{array}{c}\text { Directional } \\
\text { Asymmetry Mean (SE) }\end{array}$} & \multicolumn{3}{|c|}{ Signal-to-Noise Ratio } & \multirow{2}{*}{$\begin{array}{l}\text { Number of } \\
\text { Scan Errors }\end{array}$} & \multirow{2}{*}{$\begin{array}{c}\text { Number of } \\
\text { Outliers }\end{array}$} \\
\hline & & FA & ME & $\% \mathrm{FA}$ & & \\
\hline Thigh length & $-0.06(0.01)^{* * *}$ & 0.013 & 0.007 & $63 \%$ & 0 & 6 \\
\hline Knee height & $0.07(0.02)^{* *}$ & 0.042 & 0.008 & $85 \%$ & 1 & 5 \\
\hline Knee circumference & $0.02(0.03)$ & 0.060 & 0.018 & $77 \%$ & 0 & 0 \\
\hline Calf circumference & $0.02(0.02)$ & 0.048 & 0.002 & $96 \%$ & 1 & 12 \\
\hline Ankle circumference & $-0.25(0.15)$ & 0.74 & 2.76 & $21 \%$ & 5 & 5 \\
\hline Leg volume & $-0.04(0.85)$ & 0.734 & 0.049 & $95 \%$ & 0 & 14 \\
\hline Leg surface area & $0.447(0.893)$ & 0.767 & 0.072 & $91 \%$ & 0 & 24 \\
\hline Upper arm length & $-0.07(0.05)$ & 0.137 & 0.193 & $41 \%$ & 11 & 12 \\
\hline Forearm length & $-0.06(0.02)^{* *}$ & 0.032 & 0.011 & $74 \%$ & 0 & 3 \\
\hline Armscye circumference & $0.05(0.07)$ & 0.250 & 0.588 & $30 \%$ & 0 & 14 \\
\hline Biceps circumference & $0.01(0.04)$ & 0.100 & 0.061 & $62 \%$ & 0 & 5 \\
\hline Elbow circumference & $0.05(0.02)^{*}$ & 0.040 & 0.020 & $67 \%$ & 0 & 6 \\
\hline Wrist circumference & $-0.07(0.03)$ * & 0.055 & 0.047 & $54 \%$ & 0 & 6 \\
\hline Arm volume & $0.19(048)$ & 0.159 & 0.152 & $52 \%$ & 0 & 0 \\
\hline Arm surface area & $-0.32(0.60)$ & 0.243 & 0.258 & $48 \%$ & 0 & 4 \\
\hline
\end{tabular}


Table 2. Correlations between unsigned trait FA and trait size for all traits. Correlations between signed FA and handedness and footedness, and correlations between unsigned FA and weekly hours of symmetric and asymmetric sport activities, were provided for traits with a repeatability of $50 \%$ or more only. The trait selection on the basis of the two selection criteria (S1: repeatability above 50\%; S2: repeatability above $50 \%$ and no significant directional asymmetry) are also provided. Statistically significant correlations are indicated in bold, and the level of significance is indicated as follows: ${ }^{*} p<0.05 ;{ }^{* *} p<0.01 ;{ }^{* * *} p<0.001$.

\begin{tabular}{|c|c|c|c|c|c|c|c|}
\hline \multirow[t]{2}{*}{ Trait } & \multirow{2}{*}{$\begin{array}{c}\text { Unsigned } \\
\text { FA-Trait Size } \\
\text { Correlation }\end{array}$} & \multicolumn{2}{|c|}{$\begin{array}{c}\text { Signed FA } \\
\text { Correlations with }^{\text {a }}\end{array}$} & \multicolumn{2}{|c|}{ Unsigned FA Correlations with ${ }^{a}$} & \multirow[t]{2}{*}{ S1 } & \multirow[t]{2}{*}{ S2 } \\
\hline & & Hand. & Footed. & Asymm. Sports & Symm. Sports & & \\
\hline Thigh length & -0.14 & 0.14 & 0.05 & 0.01 & 0.09 & * & \\
\hline Knee height & 0.03 & $-0.23 *$ & -0.05 & $0.20 *$ & 0.07 & * & \\
\hline Knee circumference & 0.03 & 0.03 & -0.06 & 0.01 & -0.08 & * & * \\
\hline Calf circumference & 0.18 & -0.12 & 0.06 & 0.17 & -0.11 & * & * \\
\hline Ankle circumference & $0.48^{* * *}$ & - & - & - & - & & \\
\hline Leg volume & $0.30^{* *}$ & -0.08 & 0.01 & -0.01 & -0.08 & * & * \\
\hline Leg surface area & 0.07 & 0.12 & $0.22 *$ & 0.06 & -0.07 & * & * \\
\hline Upper arm length & 0.13 & - & - & - & - & & \\
\hline Forearm length & $0.26^{* *}$ & 0.14 & 0.06 & 0.12 & -0.14 & * & \\
\hline Armscye circumference & 0.04 & - & - & - & - & & \\
\hline Biceps circumference & 0.10 & 0.15 & -0.01 & $0.28^{* *}$ & -0.12 & * & * \\
\hline Elbow circumference & $0.32^{* * *}$ & -0.02 & 0.13 & $0.26 *$ & -0.03 & * & \\
\hline Wrist circumference & 0.05 & 0.15 & -0.05 & 0.15 & $-0.20 *$ & * & \\
\hline Arm volume & $0.19^{*}$ & $0.20 *$ & -0.07 & 0.14 & $-0.24 *$ & * & * \\
\hline Arm surface area & 0.04 & - & - & - & & & \\
\hline
\end{tabular}

Associations between the signed FA and both handedness and footedness were tested, in which the signed FA was defined as right minus left trait value, such that positive associations with handedness or footedness would indicate that more right handed or footed individuals showed a more right-biased asymmetry. In addition, associations between trait-specific unsigned FA and amount of symmetric and asymmetric sports performed were also tested. To analyze associations between average asymmetry and handedness, footedness, and hours of symmetric and asymmetric sports performed weekly, multiple regression models were fitted. Average asymmetries were calculated on the basis of (size corrected, if needed) scaled unsigned asymmetries. Four such regression models were constructed with four different response variables, namely, (i) the average asymmetry of all traits (selection criterion S1 in Table 2), (ii) the average asymmetry of all traits not showing significant DA (selection criterion S2 in Table 2), (iii) the average arm asymmetry, and (iv) the average leg asymmetry. Because for both handedness and footedness, extreme value (i.e., close to or equal to 1 and 9) indicates an extreme handedness/footedness, while 5 indicates ambidextrous or ambipedal individuals; the extremity of handedness and footedness in either direction was calculated as the absolute value of the individuals scores minus 5 .

\section{Results}

Out of the 15 traits studied, 11 showed real FA larger than ME, 5 showed significant DA, and 5 showed positive size scaling (Table 1 ). The number of outliers or scans that were unable to produce reliable scans were low ( $<5 \%$ of the total number of scans) for most traits, except calf circumference, leg volume, leg surface area, and armscye circumference, which appear to be traits that are especially prone to artefacts during the scanning (Table 1). Signed asymmetries were normally distributed (Shapiro-Wilks statistic $\mathrm{W}>0.9$ ) and kurtosis was positive for most traits and always larger than -0.2 , showing no evidence for the presence of antisymmetry.

The degree of handedness (higher score means more right-handed) correlated positively with the signed asymmetry (right-left) of arm volume and negatively with that of knee height. In addition, footedness correlated positively with the signed asymmetry of leg surface area. Amount of asymmetric 
sports correlated positively with unsigned asymmetry of knee height, biceps circumference, and elbow circumference, while amount of symmetric sports performed weekly correlated negatively with the unsigned asymmetry of wrist circumference and arm volume (Table 2). Thus, even though many tests were performed, more than 5\% were statistically significant (i.e., 8 out of $44: 19 \%$ ), and all significant associations were in the expected direction. It is thus very likely that these significant correlations reflect biologically relevant associations and not pre chance effects.

Multiple regression analyses with average asymmetry as response variable showed strongest and most consistent correlations with the number of hours of asymmetric sports per week, except for average leg asymmetry (Figure 2, Table 3). In addition, arm asymmetry also increased with footedness and decreased with hours of symmetric sports performed weekly (Figure 2, Table 3).
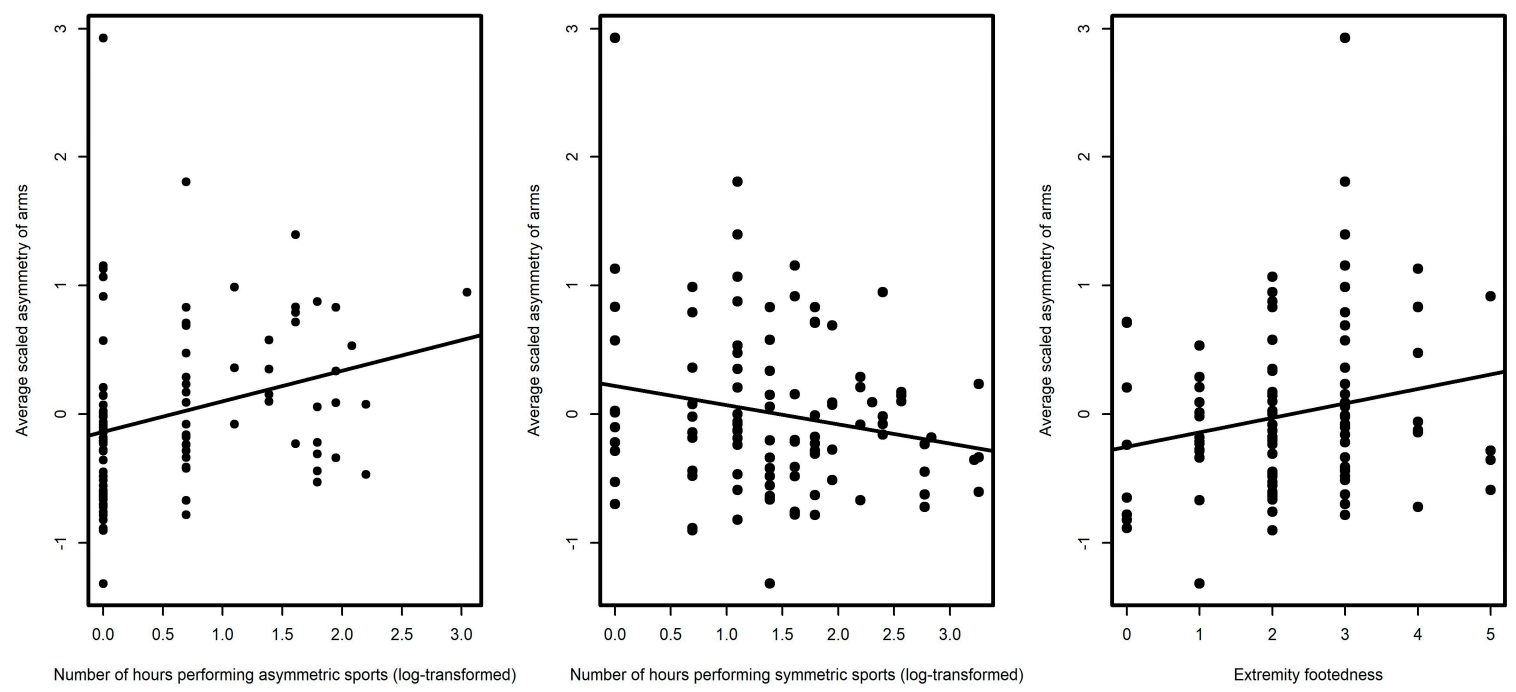

Figure 2. Associations between three measures of behavioral lateralization and asymmetry of the arms: (left) number of hours performing asymmetric sports, (middle) number of hours performing symmetric sports, and (right) extremity footedness.

Table 3. Overview of associations between average asymmetry and extremity of handedness and footedness and the amount of symmetric and asymmetric sport activity. Slopes from a multiple regression and their standard errors are provided. Statistically significant effects are indicated in bold, and the level of significance is indicated as follows: ${ }^{*} p<0.05 ;{ }^{* *} p<0.01$; ${ }^{* * *} p<0.001$. Four analyses were performed, with average asymmetry as dependent variable. Average asymmetries were calculated on the basis of all traits with a repeatability above 50\% (S1 in Table 2), all traits with a repeatability above $50 \%$ and no significant directional asymmetry (S2 in Table 2), and all arm traits and all leg traits with a repeatability above $50 \%$.

\begin{tabular}{|c|c|c|c|c|}
\hline & $\begin{array}{l}\text { Extremity of } \\
\text { Handedness }\end{array}$ & $\begin{array}{l}\text { Extremity of } \\
\text { Footedness }\end{array}$ & $\begin{array}{c}\text { Amount of } \\
\text { Asymm. Sports }\end{array}$ & $\begin{array}{c}\text { Amount of } \\
\text { Symm. Sports }\end{array}$ \\
\hline Average asymmetry 1 & $0.01(0.05)$ & $0.07(0.04)$ & $0.16(0.06) * *$ & $-0.06(0.05)$ \\
\hline Average asymmetry 2 & $-0.02(0.05)$ & $0.07(0.04)$ & $0.13(0.06) *$ & $-0.10(0.06)$ \\
\hline Average arm asymmetry & $-0.00(0.07)$ & $0.11(0.05) *$ & $0.23(0.08) * *$ & $-0.15(0.08)$ * \\
\hline Average leg asymmetry & $0.01(0.05)$ & $0.01(0.04)$ & $0.10(0.07)$ & $-0.03(0.06)$ \\
\hline
\end{tabular}

\section{Discussion}

This study confirms associations between behavioral lateralization and bodily asymmetry in arms and legs, in which effects appear strongest for the arms. This evidence comes from both single trait associations and correlations between average asymmetries and measures of differential use of both sides, in which all significant associations were in the expected directions. Correlations between the 
signed asymmetries and handedness or footedness were positive for some traits of the arms and legs, respectively. This showed that right-biased asymmetry coincided with right-biased hand or foot use. The negative correlation between the signed asymmetry of knee height and handedness is in agreement with the expectation of cross-symmetry, in which right-biased hand use results in a left-biased leg asymmetry due to compensatory mechanisms of mechanical loading [4]. Performing more asymmetric sports was associated with higher asymmetries on average and in individual traits, and was mainly associated with traits in the arm. Furthermore, symmetric sports correlated negatively with arm asymmetry, suggesting a symmetrizing effect. Interestingly, effects were also present after excluding traits that showed directional asymmetry, which means that the effects of behavioral lateralization on asymmetry are not avoided by studying traits without directional asymmetry alone.

\section{Conclusions}

All in all, even with this relatively simple quantification of behavioral lateralization on the basis of 4 questions and a merely moderate sample size, significant associations in the directions predicted were shown. This is not surprising or innovative (see e.g., also [4,5,7-9,13-16] for evidence of associations between behavioral lateralization and morphological asymmetry). The consequences, however, are crucial for the study of developmental instability in humans exhibiting strong behavioral lateralization and an enormous variation in lifestyle and behavior. On the other hand, several associations were not statistically significant. The sample size of this study does not allow one to identify traits that might not be influenced by handedness at all or at least to a lesser extent. While one could wonder if such traits would actually exist at all, further research using a global 3D approach could identify regions relatively unaffected by behavioral lateralization. Statistical correcting for behavioral lateralization does not seem to be straightforward either. As shown here, correcting for directional asymmetry or excluding traits exhibiting significant directional asymmetry did not eliminate correlations between bodily asymmetry and measures of lateralization. In conclusion, FA in some dimensions of arms and legs is likely to be confounded by behavioral lateralization. This does not mean that all traits should be discarded a priori, yet, future research should focus on parts of the human body that are not, or are only minimally, affected by behavioral lateralization such that their asymmetry does reflect developmental instability.

Conflicts of Interest: The author declares no conflict of interest.

\section{References}

1. Klingenberg, C.P. A developmental perspective on developmental instability: Theory, models, and mechanisms. In Developmental Instability: Causes and Consequences; Polak, M., Ed.; Oxford University Press: Oxford, UK, 2003; pp. 14-34.

2. Klingenberg, C.P. Analyzing fluctuating asymmetry with geometric morphometrics: Concepts, methods, and applications. Symmetry 2015, 7, 843-934. [CrossRef]

3. Palmer, A.R.; Strobeck, C. Fluctuating asymmetry: Measurement, analysis, patterns. Annu. Rev. Ecol. Syst. 1986, 17, 391-421. [CrossRef]

4. Auerbach, B.; Ruff, C.B. Limb bone bilateral asymmetry: Variability and commonality among modern humans. J. Hum. Evol. 2006, 50, 203-218. [CrossRef] [PubMed]

5. Kanchan, T.; Kumar, M.; Kumar, P.; Yoganarasimha, K. Skeletal asymmetry. J. Forensic Legal Med. 2008, 15, 177-179. [CrossRef] [PubMed]

6. Graham, J.H.; Özener, B. Fluctuating Asymmetry of Human Populations: A Review. Symmetry 2016, 8, 154. [CrossRef]

7. Daly, R.M.; Saxon, L.; Turner, C.H.; Robling, A.G.; Bass, S.L. The relationship between muscle size and bone geometry during growth and in response to exercise. Bone 2004, 34, 281-287. [CrossRef] [PubMed]

8. Özener, B. Fluctuating and directional asymmetry in young human males: Effect of heavy working condition and socioeconomic status. Am. J. Phys. Anthropol. 2010, 143, 112-120. [CrossRef] [PubMed] 
9. Van Dongen, S.; Cornille, R.; Lens, L. Sex and asymmetry in humans: What is the role of developmental instability. J. Evol. Biol. 2009, 22, 612-622. [CrossRef] [PubMed]

10. Van Dongen, S.; Gangestad, S.W. Human fluctuating asymmetry in relation to health and quality: A meta-analysis. Evol. Hum. Behav. 2011, 32, 380-398. [CrossRef]

11. Brown, W.M.; Price, M.E.; Kang, J.S.; Pound, N.; Zhou, Y.; Yu, H. Fluctuating asymmetry and preferences for sex-typical bodily characteristics. Proc. Natl. Acad. Sci. USA 2008, 105, 12938-12943. [CrossRef] [PubMed]

12. Van Dongen, S.; Molenberghs, G.; Matthysen, E. The statistical analysis of fluctuating asymmetry: REML estimation of a mixed regression model. J. Evol. Biol. 1999, 12, 94-102. [CrossRef]

13. LeMay, M. Asymmetries of the skull and handedness. Phrenology revisted. J. Neurol. Sci. 1977, 32, $243-253$. [CrossRef]

14. Plato, C.C.; Wood, J.L.; Norris, A.H. Bilateral asymmetry in bone measurements of the hand and lateral hand dominance. Am. J. Phys. Anthopol. 1980, 52, 27-31. [CrossRef] [PubMed]

15. Steele, J. Handedness in past human populations: Skeletal markers. Laterality 2000, 5, 193-220. [CrossRef] [PubMed]

16. Swissa-Sivan, A.; Simkin, A.; Leichter, I.; Nyska, A.; Nyska, M.; Statter, M.; Bivas, A.; Menczel, J.; Samueloff, S. Effect of swimming on bone growth and development in young rats. Bone Miner. 1989, 7, 91-105. [CrossRef]

(C) 2018 by the author. Licensee MDPI, Basel, Switzerland. This article is an open access article distributed under the terms and conditions of the Creative Commons Attribution (CC BY) license (http:/ / creativecommons.org/licenses/by/4.0/). 